\title{
The Stoichiometry of Binding of ATP and Its Derivatives to a Recombinant Selenophosphate Synthetase E197D Catalytically Inactive Mutant C17S
}

\author{
Yuliya V. Preobrazhenskaya ${ }^{1, ~ *, ~ A n n a ~ I . ~ S t e n ' k o 1, ~ V l a d i m i r ~ Y u . ~ L u g o v t s e v ², ~ A n d r e j ~ G . ~ M o i s e e n o k ~}{ }^{3}$, \\ Olga M. Kuratchik ${ }^{4}$, Konstantin A. Mandrik ${ }^{1}$, Alexander I. Voskoboev ${ }^{1}$ \\ ${ }^{1}$ Department of Biology and Ecology, Grodno State Kupala University, Grodno, Belarus \\ ${ }^{2}$ Food and Drug Administration, Bethesda, USA \\ ${ }^{3}$ NPTS "Biochemistry and Pharmacology", Grodno, Belarus \\ ${ }^{4}$ GR D-V Observation Station and Hospital, Grodno, Belarus
}

Email address:

Yuliya.Preabrazh@gmail.com (Y. V. Preobrazhenskaya), Vladimir.Lugovtsev@fda.hhs.gov (V. Yu. Lugovtsev)

${ }^{*}$ Corresponding author

\section{To cite this article:}

Yuliya V. Preobrazhenskaya, Anna I. Sten'ko, Vladimir Yu. Lugovtsev, Andrej G. Moiseenok, Olga M. Kuratchik, Konstantin A. Mandrik, Alexander I. Voskoboev. The Stoichiometry of Binding of ATP and Its Derivatives to a Recombinant Selenophosphate Synthetase E197D Catalytically Inactive Mutant C17S. Advances in Biochemistry. Vol. 5, No. 2, 2017, pp. 31-34. doi: 10.11648/j.ab.20170502.13

Received: December 30, 2014; Accepted: February 26, 2015; Published: April 24, 2017

\begin{abstract}
Selenophosphate synthetase (SPS) catalyses formation of the universal donor of selenium equivalents in a living cell. It performs the selenophosphate formation from ATP and selenide in ATP-dependent manner. We have checked a catalytically inactive mutant C17S of bacterial SPS from E.coli, E197D, for ATP hydrolysis and ATP-binding. The ratio obtained for ATP-binding is $9.52 \mathrm{nM}$ ATP: $7.0 \mathrm{nmol}$ enzyme, however, the fraction of the protein applied to the size-exclusive column TSK 2000 under reaction conditions was homogenious. It is likely under the ATP-binding conditions C17S mutant of SPS represents a monomer. A sequence alignment of bacterial mutant C17S from strain K12 with a human SEPHSI shows it exhibits of $31 \%$ homology. It is supposingly SPSI is a functional and structural analogue of C17S and has a similar biological activity.
\end{abstract}

Keywords: Selenophosphate Synthetase, ATP-Binding, Monomer, TNP-ATP

\section{Introduction}

Universal selenium donor in cellular processes in both prokaryotes and eukaryotes is selenophosphate which formation is catalyzed by selenophosphate synthetase (SPS) in bacteria - a gene product of selD [1]. This ATP-dependent protein performs the conversion reaction of ATP and selenide to selenophosphate, AMP and orthophosphate in a stoichiometric ratio of $1: 1: 1[2,3]$ :

$$
\mathrm{ATP}+\mathrm{HSe}-+\mathrm{H}_{2} \mathrm{O} \rightarrow \mathrm{H}_{2} \mathrm{SePO}_{3^{-}}+\mathrm{AMP}+\mathrm{Pi} .
$$

Previously, it was shown that the SPS exhibits ATPase activity in the absence of selenium ions in the medium and the final products of the reaction - AMP and Pi - coincide with those in the reaction proceeding with selenide [2]. The $1^{\text {st }}$ step in the reaction mechanism is a nucleophylic attack on the $\gamma$-phosphoryl group of ATP as presented in [4]. The phosphoryl-enzyme intermediate then undergoes nucleophylic attack by selenide to form selenophosphate and free enzyme.

In the present work we would like to present an oligomeric state of SPS catalytically inactive mutant during the reaction conditions giving insight to the catalytic mechanism of the overall reaction. For that, we have used the data both on the ATP-binding and ATP hydrolysis. We demonstrate on the mutant form of the SPS protein, C17S, which is not able to proceed the second step of the SPS reaction but still able to bind ATP. 


\section{Experimental}

\subsection{Enzyme Preparation}

We used a homogenous preparation of selenophosphate synthetase (SPS) obtained after immunoaffinity chromatography step (Fig 1). The observed band corresponds to a protein of $\mathrm{Mr} \sim 38 \mathrm{kDa}$, which is consistent with earlier data for SPS from E.coli.

\subsection{Strains and Plasmids}

An expression system for $E$. coli SPS was created based on plasmids for overexpression of pET11a (Stratagene, USA) as it is described in [5]. A wild-type and mutated SelD gene were cloned into the plasmid in order E.coli BL21 (DE3) strain to be transformed with it. After cloning of PCR products, ligating them with $\mathrm{pET} 11 \mathrm{a}$ vector, digesting with rectriction endonucleases and inserting into plasmid for overexpression we have obtained a wild-type SelD protein with synonymic substitution E197D and mutant enzyme C17S of SPS, that was confirmed by sequencing. Primers for PCR contained sites of recognition for NdeI and BamH1. The mutant recombinant enzyme $\mathrm{C} 17 \mathrm{~S}$ was obtained by sitedirected mutagenesis of SelD gene according to the manufacturer instructions (Quick Change ${ }^{\mathrm{TM}}$ Site-Directed Mutagenesis Kit, Stratagene, USA). The recombinant mutant obtained does contain its own SPS gene, SelD, in contrary to the strain MB08 used as a host in [6] which was SelDdeficient.

\subsection{Purification of SELD Protein}

E. coli BL21 (DE3) strain transformed with the plasmid pET11a (Stratagene, USA) containing an ORF of SelD gene from BL-21 Gold was grown in 500-ml flasks with rotation at $150 \mathrm{rpm}$ in LB medium with ampicillin $50 \mu \mathrm{g} / \mathrm{ml}$. After the cell density reached an $\mathrm{A}_{590}=0.6$, the $S e l D$ gene was induced by adding $0.4 \mathrm{mM}$ isopropyl- $\beta-D$-thiogalactopyranoside (Sigma, USA), and the fermentation was continued for $3 \mathrm{~h}$.

It was determined after cell separation from the cultural broth, disruption on the ultrasonic disintegrator UD-20 (Poland) and centrifugation at $18000 \mathrm{rpm}$ during $20 \mathrm{~min}$ in order to remove cell debris, $95-97 \%$ of recombinant protein was located in a soluble part of the extract. Further purification of the supernatant containing wt or mutant enzymes was performed by ammonium sulfate precipitation, DNAse (Sigma, USA) was added on the stage of cell disruption as well as PMSF, therefore, the supernatant did not contain any traces of DNA. The protein sought was precipitated from 0 to $55 \%$, after that fraction collection and desalting it on the size-exclusive column of Sephadex G-10 it was applied onto an ion-exchange DEAE-Sephadex A-50 column according to the procedure developed by us in [5]. Such a method of purification and isolation allowed to obtain $20 \mathrm{mg}$ of soluble protein per 1 liter of bacterial culture. The isolated proteins were monitored for purity by electrophoresis on SDS-PAGE and verified by Western blotting (Fig.1). As primary antibodies chicken anti-SelD IgG $(1: 1000)$ were used, as secondary ones - anti-chicken rabbit IgG (1:10000). Protein bands on the nitrocellulose membranes were visualized with 3, 3"- diaminobenzidin and photographed or quantified.

\subsection{Preparation of SPS Antibody Affinity Column}

Purified SPS, $10 \mathrm{mg}$, was coupled to $10 \mathrm{ml}$ of resuspended CNBr-activated Sepharose-4B as described by the manufacturer. (Amersham Biosciences, Uppsala, Sweden). The gel was pre-equilibrated at $\mathrm{pH} 7.4$ in Tris buffer and a small-size FPLC column was packed with it (bed-size $0.5 \times 10$ $\mathrm{cm})$.

\subsection{ATPase Activity Determination}

ATPase activity of SPS was determined in the absence of selenium in the reaction mixture. The reaction was performed in $0.1 \mathrm{M} \mathrm{K}$-Tricine buffer at $37^{\circ} \mathrm{C}$ and $37 \mu \mathrm{M}$ enzyme concentrations. The reaction mixture was heated to $95^{\circ} \mathrm{C}$, put on ice and filtered prior to application on the HPLC column. Distillation of the resulting nucleotides were performed on a reverse phase column of $\mathrm{C} 18$, using HPLC equipment of the company Agilent 1100. In our improved methodology of nucleotides separation with acetonitrile (linear gradient of 0 $\rightarrow 5 \%$ ) a peak of AMP as the final reaction product is well separated from other peaks, and the remainder of the substrate consisting of ADP and ATP (Figure 2).

\section{Results}

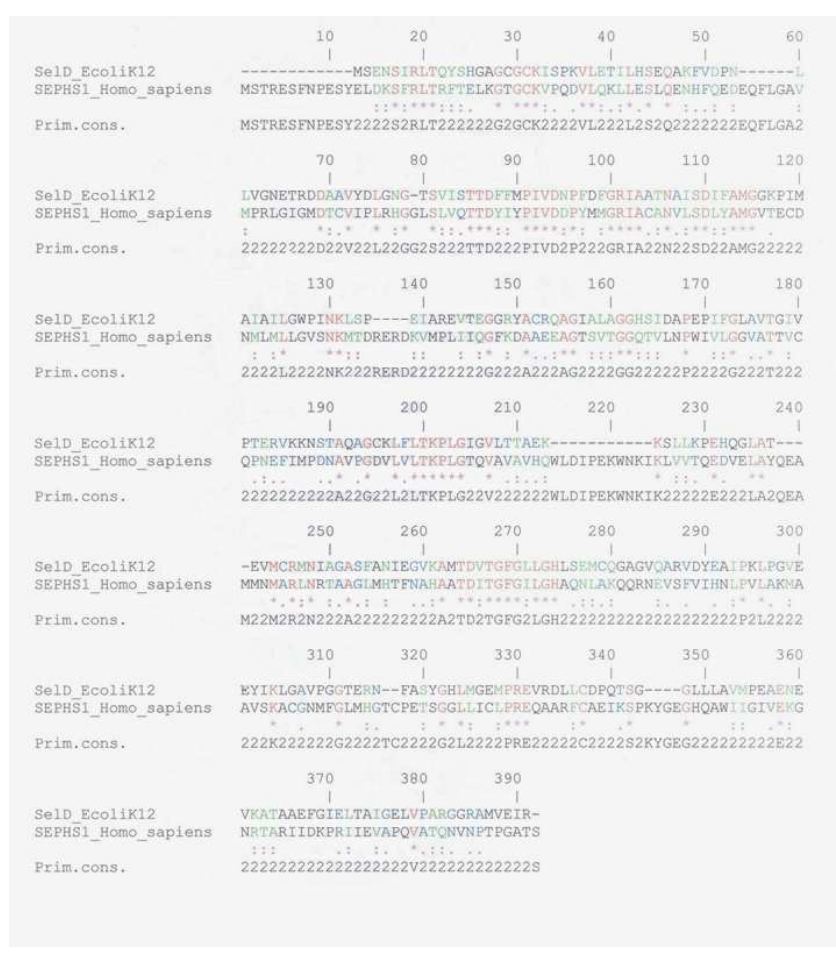

Scheme 1. Alignment of C17S mutant of E. coli and a human SPSI. 


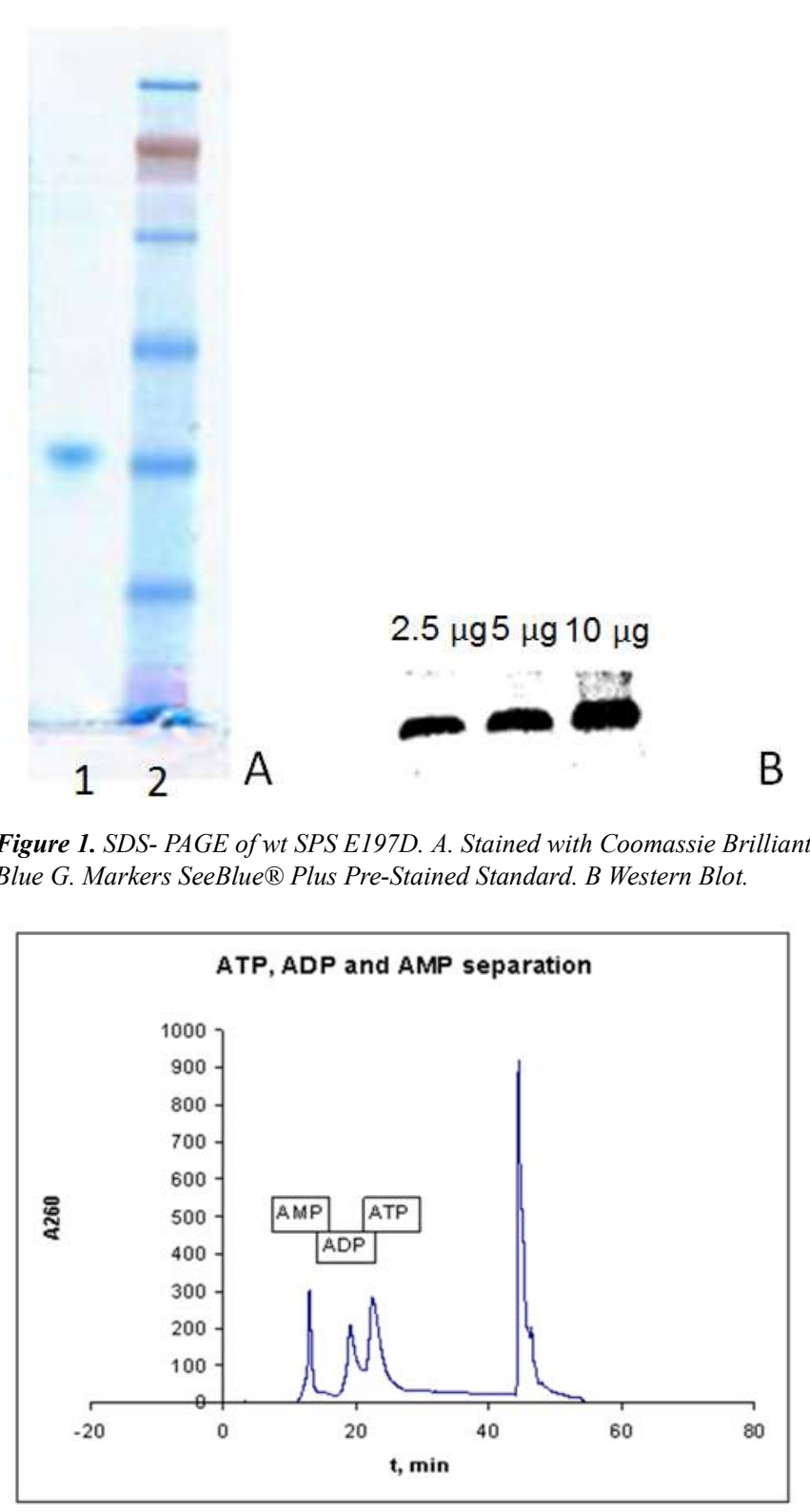

Figure 2. Chromatogram of distillation nucleotides ATP, ADP and AMP by HPLC (Agilent 1100).

\subsection{ATP Hydrolysis Reaction Studies}

In Fig. 2 a peak of ADP indicates that there might be an intermediate stage of the reaction with $\mathrm{ADP}$ as an intermediate. As far as no ADP is detected as a final product, a subsequent hydrolysis of ADP takes place. Calibration curve by AMP is a linear dependence of the ATPase activity of the enzyme on the concentration of the product, it means that the activity can be determined by AMP formation (Figure 3). Enzyme activity of wild type SPS E197D determined in these conditions was $34 \mathrm{nmol} /(\mathrm{min} * \mathrm{mg}$ ).

\subsection{TNP-ATP Binding Studies}

The presence of competitive replacement of ATP with a fluorescent derivative of ATP, TNP-ATP, in the active site of selenophosphate synthetase with $\mathrm{IC}_{50}=46 \mathrm{mM}$, suggesting the possibility of its use for determining the amount of the ATP-binding sites of the enzyme was revealed. We have investigated the binding stoichiometry of ATP molecule to SPS using fluorescent derivative of ATP, TNP-ATP and recombinant SPS with one synonymous substitution, the wild-type, E197D [5]. The final value of the ratio proved to be 2: 1 ( $\mathrm{mol} / \mathrm{mol})$ substrate: enzyme, respectively, was obtained from graphical linearized plot in Scatchard graph (not shown).

It is known that a mutant SPS C17S is catalytically inactive and is only capable of ATP hydrolysis to AMP [6]. The created based on the same plasmid construct, this mutant showed the ability to bind TNP-ATP 1: 1 ( $\mathrm{mol} / \mathrm{mol})$.

\subsection{Alignment of SPS and Human SEPHS1}

From the alignment of mutant $\mathrm{C} 17 \mathrm{~S}$ from bacterial strain K12 and human SPSI (Scheme) it can be seen that after removing "cap" a bacterial SELD product that carries a point mutation in 17 position reminds a human one of SPS homologues, namely SPS I , that apparently has Thre residue in that position as well. We have found a structural homologue of SPSI that exhibits, according to this alignment, made with Clustal W blast program, of $31 \%$ homology $(22 \%$ absolutely identical), that is quite enough to predict human SPSI and bacterial catalytically inactive mutant that retains only $1 / 3$ of wt ATPase activity could be possibly functional analogues and may play a similar biological role. This hypothesis was already known, but not proved by experimental data [7]. It appears, we are the first who has found the direct proof for that. Dr. Gladyshev and group have showed in 2006 [8] and then in 2011 [9] human SPSI is distinct from SPSII as far as definitely does not bind selenium and could not make selenophosphate. From our alignment it could be seen a glycine-rich putative region corresponding to an ATP-binding site of kinases and some phosphatases situated in position $265-275$ is absolutely identical in E.coli SPS and in human SPSI as well. We suppose that SPSI has ATP-binding properties and may function as protein kinase.

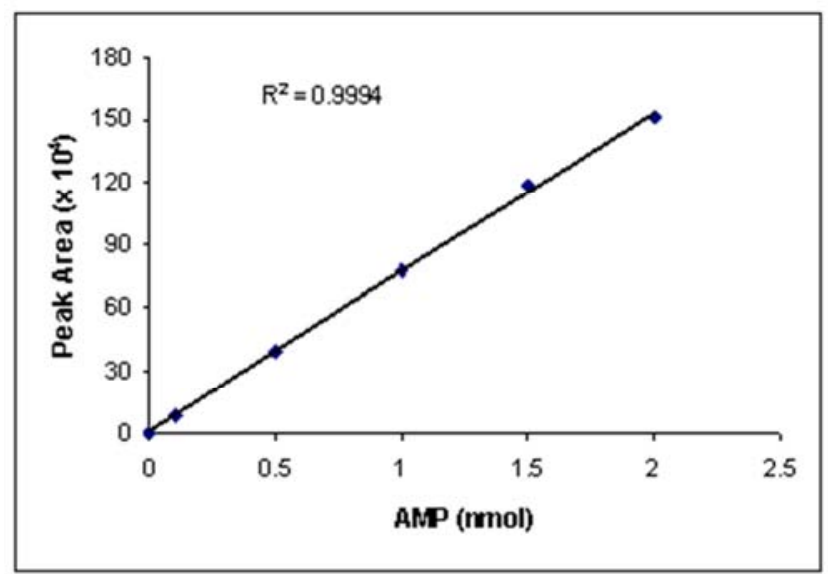

Figure 3. A calibration curve for determining the number of AMP after separation by HPLC. 


\subsection{Interplay Between Structure and Function}

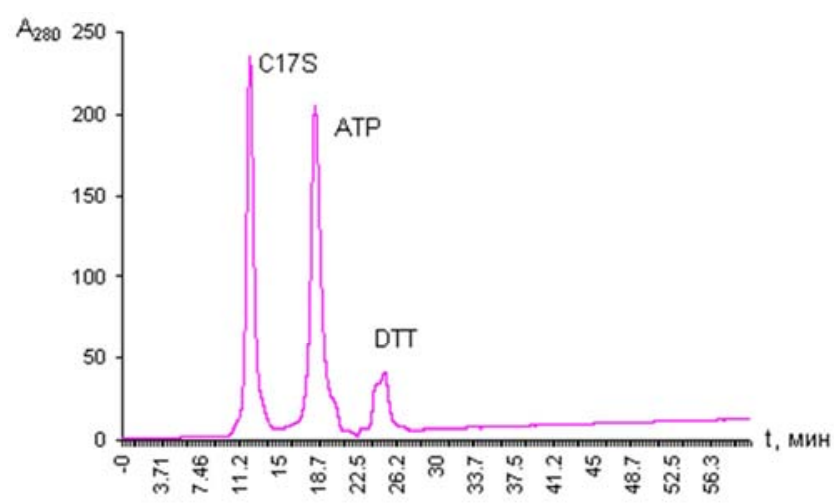

Figure 4. Analytical gel-filtration by HPLC method .Column TSK - S2000 SW. 1,-protein, 2-ATP, 3 -dithiothreitol.

By analytical gel filtration monodispersity (uniformity) of the fraction of a native recombinant SPS and catalytically inactive mutant form $\mathrm{C} 17 \mathrm{~S}$ of the enzyme on a HPLC column TSK - S2000 SW was investigated. When this reaction is carried out with the substrate binding of ATP to control the reactivity, and thus the functional conformation of a protein molecule, the corresponding protein before application to the column was incubated with $\left[{ }^{14} \mathrm{CATP}\right]$ for $10 \mathrm{~min}$. The binding reaction is conducted in a medium of $100 \mathrm{mM}$ Tricine-KOH, pH 8.0, $30 \mathrm{mM} \mathrm{KCl}, 0.5 \mathrm{mM}$ dithiothreitol (DTT), $4 \mathrm{mM} \mathrm{MnCl} \mathrm{M}_{2}$. If for the native SPS in these reaction conditions we obtained protein peak separation into two fractions, which may indicate the heterogeneity of the SPS under the reaction conditions, then for its mutant $\mathrm{C} 17 \mathrm{~S}$, as it is seen from the chromatogram (Figure 4), the protein peak is quite homogenous. The mutant protein $\mathrm{C} 17 \mathrm{~S}$ incapable of catalyzing the reaction of the main SPS activity - selenophosphate formation, binds the substrate ATP in a ratio $=9.52 \mathrm{nM}$ ATP: $7.0 \mathrm{nmol}$ enzyme. Moreover, there is an experimental evidence for the wild type of SPS protein to form $\gamma$-phosphoryl-enzyme intermediate demonstrated by positional isotope exchange from the $\beta, \gamma$-bridge oxygen -18 of $\left[\gamma^{18} \mathrm{O}_{4}\right]$ ATP into the $\beta$-nonbridge position [4]. However, no positional isotope exchange was observed with $\mathrm{C} 17 \mathrm{~S}$ mutant. In this regard, given in [10] example of $\mathrm{C} 17 \mathrm{~S}$ crystallizing in the form of a dimer, very illustrative for the crystallography data of the protein, obviously could be applied only to a crystalline form of the mutant SPS. Under the ATP-binding reaction conditions, a functionally inactive mutant $\mathrm{C} 17 \mathrm{~S}$ that still retains about $12 \%$ of ATPase activity according to our data is likely a monomer. In this regard, the need for further study of the relationship between function and the conformation of the SPS is highly desirable.

\section{References}

[1] Hirosava-Takamori M., Jaecle H., Vorbruggen G. (2000). EMBO reports, 1, 441-446.

[2] Veres, Z., Kim, I. Y., Scholz, T. D., and Stadtman, T.C (1994) J. Biol. Chem., 269, 10597-10603.

[3] Lacourciere, G. M., Mihara, H., Kurihara, T., Esaki, N., and Stadtman, T.C. (2000) J. Biol. Chem., 275, 23769-23773.

[4] Mullins, L. S., et al (1997) J. Am.Chem.Soc., 119, 6684-6685.

[5] Preobrazhenskaya Y. V., Stenko A. I., Shvarts M. V., Lugovtsev V. Yu. (2013) J. Amino Acids, 2013.

[6] Kim, I. Y., Veres, Z., Stadtman, T. C. (1992) J. Biol. Chem., 267, 19650-19654.

[7] Low, S. C., Harney, J. W., Berry, M. J. (1995) J. Biol. Chem., 270, 21659-21664.

[8] Xu, Xue-M., Carlson, B. A, Mix, H., Irons R., Berry, M. J, Gladyshev V. N and Hatfield D.L. FASEB J. March 200620 (Meeting Abstract Supplement) A428.

[9] Selenium: Its Molecular Biology and Role in Human Health by Dolph L. Hatfield, Marla J. Berry, Vadim N. Gladyshev, Springer Science \& Business media, 2011, p.27.

[10] Noinaj N., et al. (2012) J. Bacteriol., 194, 499-508. 